\title{
Absence of pneumococcal PcsB is associated with overexpression of LysM domain-containing proteins
}

\section{Correspondence \\ Eszter Nagy \\ eszter.nagy@arsanis.com \\ Carmen Giefing-Kröll \\ CGiefing@intercell.com}

Received 5 September 2010

Revised 15 March 2011

Accepted 5 April 2011

\author{
Carmen Giefing-Kröll, ${ }^{1}$ Kira E. Jelencsics, ${ }^{1}$ Siegfried Reipert ${ }^{2}$ \\ and Eszter Nagy ${ }^{1} \dagger$
}

\author{
${ }^{1}$ Intercell AG, Vienna Biocenter 3, 1030 Vienna, Austria \\ ${ }^{2}$ Max F. Perutz Laboratories, Dr. Bohrgasse 9/5, 1030 Vienna, Austria
}

\section{INTRODUCTION}

The pneumococcus is one of the most important human pathogens, and defeats its host mainly by its fast multiplication and the tissue damage induced by inflammatory responses. A better understanding of the critical and essential steps in pneumococcal cell division and multiplication is important in order to develop novel strategies to reduce the high mortality associated with invasive pneumococcal infections.

As a crucial stress-bearing and shape-maintaining element, the integrity of the bacterial cell wall is essential to cell viability. Peptidoglycan, a cross-linked polymer of glycan strands and peptide chains, forms the scaffold of this structure. To maintain the protective nature of the cell

tPresent address: Arsanis Inc., Campus Vienna Biocenter 4, A-1030 Vienna, Austria.

Abbreviations: $\mathrm{CHAP}$, cysteine, histidine-dependent amidohydrolase/ peptidase; $C_{T}$, cycle threshold; DAPI, 4,6-diamidino-2-phenylindole; qRT-PCR, quantitative real-time PCR; RBB, Remazol brilliant blue. wall, bacterial cell division requires a tightly regulated and complex interplay of topological processes, biosynthetic reactions and cleavage mechanisms. In ovococcus bacteria such as Streptococcus pneumoniae, cell division proceeds along successive parallel planes perpendicular to the long axis of the cell. This mode of division contrasts with that of spherical coccus species such as Staphylococcus aureus, which divide in alternating perpendicular planes reflected by the mode of peptidoglycan synthesis in these species (for a review, see Zapun et al., 2008).

In ovococcus species, peptidoglycan is synthesized at the septum and at the equatorial rings by the insertion of peptide-carrying disaccharide units into the existing murein sacculus through transglycosylation and transpeptidation, whereas spherical species have only septal peptidoglycan synthesis (for a review, see Zapun et al., 2008). Cell division is completed by the action of murein hydrolases cleaving the peptidoglycan at the septum and thus releasing the adjacent daughter cells. Hydrolases are important for cell wall growth and separation during cell division, peptidoglycan recycling and turnover. These 
enzymes also participate in autolysis under certain growth conditions, and contribute to bacterial pathogenicity and susceptibility to antibiotics (Höltje \& Tuomanen, 1991; Höltje, 1995; Vollmer et al., 2008).

PcsB of S. pneumoniae (annotated as SP2216 in the TIGR4 genome) is a predicted murein hydrolase, and has orthologues in several streptococcal species that have been characterized to a varying degree, e.g. GbpB in Streptococcus mutans (Chia et al., 2001a, b; Mattos-Graner et al., 2001, 2006) and PcsB in Streptococcus agalactiae (Reinscheid et al., 2001, 2003). PcsB and its orthologues consist of four major parts: a signal peptide, an N-terminal coiled-coil domain containing leucine-zipper motifs, an alanine-rich region of variable length and a C-terminal cysteine, histidine-dependent amidohydrolase/peptidase (CHAP) domain.

Orthologues of PcsB in several species have been described as immunodominant antigens during human infection and confer protection in animal models (Chia et al., 2001a; Giefing et al., 2008; Smith et al., 1994, 2001; McIver et al., 1996; Teng et al., 2003). The pneumococcal PcsB has also been shown to be a highly immunogenic protein in humans and a protective antigen in animal models in our laboratory (Giefing et al., 2008). GbpB in S. mutans and PcsB in S. agalactiae have been found to be important, but non-essential for growth. Gene deletion mutant strains display greatly reduced growth rates and altered cell morphology, with multiple and misplaced division septa within a single cell (Chia et al., 2001a; Reinscheid et al., 2001). Although pneumococcal PcsB has been described as being essential for S. pneumoniae strains D39 and R6 (Barendt et al., 2009; Ng et al., 2003, 2004, 2005), we reported previously that we were able to generate $p c s B$ null strains in two different genetic backgrounds, and found similar phenotypic defects to those previously described for its streptococcal homologues (Giefing et al., 2008).

The CHAP domain is found in proteins from bacteria, bacteriophages, archaea and eukaryotes, and is commonly associated with domains, such as the bacterial-type $\mathrm{SH} 3$ and amidase domains, that have been shown to be involved in peptidoglycan hydrolysis (Bateman \& Rawlings, 2003; Rigden et al., 2003). The CHAP domain itself has peptidoglycan lytic activity, and conserved cysteine and histidine residues, which have been found to be required for its function, are also present and essential in pneumococcal PcsB (Ng et al., 2004). Some of the CHAP-domain proteins exert robust hydrolase activity also in vitro, e.g. Iap from Listeria monocytogenes (Wuenscher et al., 1993), whereas PcsB and its orthologues have failed to show murein hydrolase activity in biochemical assays (Mattos-Graner et al., 2006; Reinscheid et al., 2001, 2003). Similarly, analysis of the muropeptide composition in gene deletion or low-expressing strains has not revealed any differences between wild-type and mutant strains (Reinscheid et al., 2003; Barendt et al., 2009).

PcsB and its homologues in other streptococcal, enterococcal and lactococcal species share a similar organization of the genome region, namely they localize in the immediate vicinity of the $m r e C$ and $m r e D$ genes, which encode the homologues of morphogenic proteins in rod-shaped bacteria (Stewart, 2005; Osborn \& Rothfield, 2007), pointing towards a possible involvement of PcsB in the determination of cell morphology. The membrane-spanning essential protein $\mathrm{MreC}$ has been shown to link the bacterial cytoskeleton to a variety of cell wall-modifying enzymes. Additionally, MreC can form filaments that run perpendicularly to the membrane (Divakaruni et al., 2007; Kyburz et al., 2010). However, it has been shown that perturbations of expression of the upstream mre $C D$ genes do not contribute to the cell division defects of $p c s B$ mutants (Barendt et al., 2009).

As the specific function of $\mathrm{PcsB}$ and its homologues remains unknown to date, we aimed to contribute to a better understanding of the role of pneumococcal PcsB in the course of this study. We show that PcsB is not essential for in vitro viability in four different genetic backgrounds, and that the phenotypic defects of $p c s B$ deletion strains can be restored by extrachromosomal expression. In addition we demonstrate that PcsB localizes at the cell poles, but is excluded from the division septum. The most obvious changes in gene transcription in the pcsB null mutant cells are associated with highly increased expression of LysM domain-containing proteins, indicating a similar or overlapping function shared by these proteins.

\section{METHODS}

Bacterial strains and growth conditions. TIGR4 (serotype 4) and PJ1324 (serotype 6B) strains were provided by Birgitta HenriquesNormark (Swedish Institute for Infectious Disease control), EF3030 (serotype 19F) by David Briles (University of Alabama) and 4DS234194 (serotype 4) strain by Eddie Ades [Centers for Disease Control (CDC), USA]. $\triangle p c s B$ and $\triangle \mathrm{SP} 2063$ mutant strains were generated as described previously (Giefing et al., 2008). Mutant strains were verified first by PCR amplification of the altered genomic region using primer combinations specific for flanking and integrated fragments. Southern blot analysis confirmed integration into the expected chromosomal region, and Western blotting proved the absence of protein expression. Northern blotting was performed to confirm the absence of target gene mRNA and the presence of flanking mRNA transcripts. Bacteria were cultured in Todd-Hewitt broth supplemented with $0.5 \%$ yeast extract (THY), including the appropriate antibiotic for mutant strains at $37{ }^{\circ} \mathrm{C}$ in an atmosphere of $5 \% \mathrm{CO}_{2}$.

Complementation of the pcsB deletion strain. For complementation, extrachromosomal expression of PcsB from a plasmid was chosen. Briefly the complete $p c s B$ gene was cloned into the multiple cloning site of pMU1328 with a fucose-inducible promoter (Chan et al., 2003) via EcoRI and XbaI. The plasmid was transformed as described previously (Giefing et al., 2008), and erythromycin- and kanamycin-resistant clones were selected for further analysis. Empty plasmid alone is abbreviated as pMU, the one containing $p \operatorname{cs} B$ as pMU-PcsB throughout the text.

Antibiotic sensitivity testing. Pneumococci were grown until they reached $\mathrm{OD}_{620} \sim 0.5$. Subsequently, $2 \times 10^{7}$ bacteria were streaked on a blood agar plate to ensure a dense bacterial lawn and after $15 \mathrm{~min}$ an 
antibiotic test disc (Fluka and bioMérieux) was placed into the centre of each plate. Plates were incubated at $37{ }^{\circ} \mathrm{C}$ and $5 \% \mathrm{CO}_{2}$ for $24 \mathrm{~h}$, and the diameters of growth inhibition zones were measured. A mean value was calculated from three independent experiments and used to express the percentage increase in antibiotic sensitivity relative to the respective wild-type strain.

Cloning and expression of recombinant pneumococcal proteins. The following PcsB constructs were generated from TIGR4 genomic DNA: amino acids 28-392 (PcsB-FL), amino acids 28-278 (PcsB-N) and amino acids 258-392 (PcsB-CHAP) (see also Fig. 4a). FtsZ (amino acids 2-419) and PspA (SP0117) were generated in previous studies (Giefing et al., 2008, 2010). All genes were cloned into the pET28b $(+)$ vector (Novagen) and expressed with a Cterminal His-tag in Escherichia coli BL21 Star (Invitrogen). Proteins were bound to Ni-Sepharose beads (GE Healthcare) and eluted with $500 \mathrm{mM}$ imidazole in $20 \mathrm{mM} \mathrm{NaH} \mathrm{PO}_{4}, 0.5 \mathrm{mM} \mathrm{NaCl}, \mathrm{pH}$ 7.4. Proteins were extracted from inclusion bodies that were solubilized in $8 \mathrm{M}$ urea in $50 \mathrm{mM}$ Tris/ $\mathrm{HCl}, \mathrm{pH} 8$, and gradually dialysed against decreasing concentrations of urea-containing Tris buffer and $\beta$ mercaptoethanol. Insoluble proteins were removed by centrifugation and the protein solution was prepared in Tris buffer at $1-2 \mathrm{mg} \mathrm{ml}^{-1}$. The purity of the proteins used for interaction studies was $>90 \%$ based on SDS-PAGE (Fig. 4b).

Generation of hyperimmune mouse and rabbit sera. $\mathrm{C} 3 \mathrm{H} /$ HeNHsd mice (10 mice per group, female, 6-10 weeks, Harlan Winkelmann) were immunized three times at 14 day intervals subcutaneously (into the flank) with $50 \mu \mathrm{g}$ recombinant protein (FtsZ, PcsB-FL, PcsB-N, PcsB-CHAP, SP0107, SP0082, SP0564, SP0498, MalX or PspA) and hyperimmune sera were collected on day 35. New Zealand White rabbits (Charles River) were immunized at days $0,28,42$ and 56 with $250 \mu \mathrm{g}$ PcsB-N, StkP-C or PsaA recombinant protein, and complete Freund's adjuvant (CFA)/ incomplete Freund's adjuvant (IFA) as adjuvant, and terminally bled on day 70 .

Electron microscopy. Wild-type, $\Delta p c s B$ and extra-chromosomally complemented mutant cells of $S$. pneumoniae strains were fixed with $2.5 \%$ glutaraldehyde in Dulbecco's PBS (Gibco) ( $\mathrm{pH}$ 7.4) for $2 \mathrm{~h}$ on ice and for $1 \mathrm{~h}$ at room temperature, transferred into cellulose capillary tubes $\left(0.2 \mathrm{~mm}\right.$ diameter), fixed with $2 \% \mathrm{OsO}_{4}$ in Sorensen's phosphate buffer (0.1 M sodium phosphate, $\mathrm{pH} 7.4)$ for $90 \mathrm{~min}$ and dehydrated with increasing concentrations of ethanol. Samples were embedded in epoxy resin (Agar 100), and thin sections (60-80 nm) were cut with a Leica Ultracut $S$ ultramicrotome, mounted on copper grids, contrasted by uranyl acetate and lead citrate, and examined at $80 \mathrm{kV}$ in a JEOL JEM-1210 electron microscope. Images were acquired using a digital camera (Morada) for the wide-angle port of the transmission electron microscope and with analySIS FIVE software (Soft Image System).

Western blotting. Proteins were separated on 4-20\% gradient SDSPAGE gels under reducing conditions and blotted to nitrocellulose membranes using a dry-blotting system (Invitrogen). Blocking and incubation with primary $(1: 1000$ dilution) and horseradish peroxidase (HRP)-conjugated secondary antibodies ( $1: 5000$ dilution) were done in $5 \%$ milk in PBS containing $0.1 \%$ Tween 20 for $1 \mathrm{~h}$ at room temperature. Membranes were imaged with chemiluminescent substrate (Chemiglow, Alpha Innotech) and a charge-coupled device (CCD) camera (FluorChem SP, Alpha Innotech).

Immunofluorescence microscopy. Pneumococci were stained as described previously (Giefing et al., 2010). Briefly, bacteria were fixed with $3 \%$ paraformaldehyde, transferred to polylysine-coated microscope slides and permeabilized with $0.1 \%$ Triton X-100 in PBS. After blocking, primary antibodies were added at a 1:50 dilution, followed by incubation with anti-rabbit-Cy2 and anti-mouse-Texas Red antibodies (JacksonImmuno) and staining with 4,6-diamidino-2phenylindole (DAPI). Slides were mounted with Eukitt (Fluka) and visualized with a Zeiss Axiovert 200M microscope with a Zeiss LSM510META confocal laser-scanning unit and an $\alpha$ Plan-Fluor $\times 1001.45$ oil (aperture, $0.11 \mathrm{~mm}$ ) objective (Zeiss). Images were prepared with Zeiss LSM Image Browser version 4.2.0.121 and Adobe Photoshop CS4 software by adjusting the brightness and contrast of the staining.

Determination of cellular DNA content by flow cytometry. Pneumococci in different growth stages were fixed with $70 \%$ ethanol. After washing with PBS, bacteria were incubated in staining solution (0.2 mg DNase-free RNase $\mathrm{A} \mathrm{ml}^{-1}, 20 \mu \mathrm{g}$ propidium iodide $\mathrm{ml}^{-1}$ and $0.1 \%$ Triton $\mathrm{X}-100$ in PBS) for $30 \mathrm{~min}$ at room temperature. Samples were measured with a flow cytometer (FC500, Beckman Coulter) and data were analysed using CXP software (Beckman Coulter).

In-gel peptidoglycan lysis assay. The peptidoglycan lytic activity of PcsB was tested in a zymogram assay according to Lepeuple et al. (1998). Total bacterial lysate and culture supernatant of TIGR4 wildtype and recombinant PcsB-FL were analysed on a $12 \%$ polyacrylamide gel containing $0.5 \%$ SDS-treated pneumococcal cells. Cells were prepared by heating at $100{ }^{\circ} \mathrm{C}$ for $30 \mathrm{~min}$ in $4 \%$ SDS according to the method of Schubert et al. (2000). After electrophoresis, the 'cell-gel' was renatured in $0.1 \%$ Triton X-100 in 50 mM MES-NaOH, $\mathrm{pH} 6.0$, at $37{ }^{\circ} \mathrm{C}$ for $48 \mathrm{~h}$. Gels were stained with $0.1 \%$ methylene blue in $0.01 \% \mathrm{KOH}$ and destained with water. Bacteriolytic activity was visible as clear bands on a blue background.

Peptidoglycan isolation and dye-release assay. Pneumococcal cell wall was isolated as described previously (Zhou et al., 1988). Briefly, TIGR4 and PJ1324 cells were killed by autoclaving, resuspended in $10 \% \mathrm{TCA}$ and incubated at $4{ }^{\circ} \mathrm{C}$ overnight. Insoluble material was collected, heated in 5\% TCA for $10 \mathrm{~min}$ and trypsinized overnight at $37^{\circ} \mathrm{C}$, followed by heat-inactivation. After extensive washing with PBS, the purified cell wall material was stored at $-20{ }^{\circ} \mathrm{C}$.

Remazol brilliant blue (RBB) derivatives were prepared by incubating insoluble peptidoglycan in $0.02 \mathrm{M} \mathrm{RBB}$ in $0.25 \mathrm{M} \mathrm{NaOH}$ for $6 \mathrm{~h}$ at $37^{\circ} \mathrm{C}$ and $12 \mathrm{~h}$ at $4{ }^{\circ} \mathrm{C}$. Dyed products were washed extensively with PBS until the supernatant was colourless (Zhou et al., 1988). A $1 \mathrm{ml}$ volume of peptidoglycan solution corresponded to the amount isolated from $10^{10}$ bacteria.

For dye-release assays, $100 \mu \mathrm{g}$ recombinant PcsB protein (full-length, $\mathrm{N}$-terminal and C-terminal CHAP domain) was incubated with $900 \mu \mathrm{l}$ peptidoglycan solution in a total volume of $1 \mathrm{ml}$ at $37{ }^{\circ} \mathrm{C}$ overnight, then the mixture was separated by centrifugation for $1 \mathrm{~min}$ at $10000 \mathrm{~g}$ and the release of blue dye to the supernatant was measured at $595 \mathrm{~nm}$.

RNA isolation. Bacteria grown in THY medium were harvested in the exponential phase $\left(\mathrm{OD}_{620} \sim 0.4\right)$ and total RNA was isolated using the RNeasy Midi kit (Qiagen) according to the manufacturer's instructions, performing on-column DNase digestion using the RNase-free DNase set (Qiagen). The integrity and purity of the RNA were verified by agarose gel electrophoresis, determining $\mathrm{OD}_{260}: \mathrm{OD}_{280}$ ratios and performing RT-PCR using the Superscript III First-Strand Synthesis system (Invitrogen) and random hexamers in the standard protocol.

Analysis of changes in mRNA levels. Microarray analysis was performed at Eurogentec (Seraing, Belgium) using Cy3- and Cy5labelled samples on an S. pneumoniae MicroArray [Eurogentec, 2085 ORFs of S. pneumoniae (TIGR4)], including data processing and 
normalization (S. pneumoniae Hybridization Service package). Based on these data, genes that showed changes of at least twofold in mRNA expression were selected for quantitative real time-PCR (qRT-PCR).

qRT-PCR analyses were performed using the LightCycler 480 SYBR Green I Master system (Roche). The mRNA expression of the selected genes was analysed in three independent RNA isolations from TIGR4 and PJ1324 wild-type and isogenic $\Delta p c s B$ strains using gene-specific internal primers. Quantitative differences for each sample were determined using the $2-\left(\Delta C_{\mathrm{T}}^{\text {sample }}-\Delta C_{\mathrm{T}}^{\text {calibrator }}\right)$ method (Livak \& Schmittgen, 2001), where $C_{\mathrm{T}}$ is the cycle threshold.

\section{RESULTS}

\section{PcsB is not essential for in vitro viability of $S$. pneumoniae, and phenotypic changes can be restored by complementation of PcsB}

Pneumococcal $p c s B$ has been reported to be an essential gene in the R6 and D39 strains (Barendt et al., 2009; Ng et al., 2003, 2004, 2005). However, we were able to generate $p c s B$ null mutants in the genetic background of TIGR4 (a serotype 4 strain) and a clinical isolate of a 6B strain, both capable of inducing lethal sepsis in mice (Giefing et al., 2008). During this study we generated viable $\Delta p c s B$ mutants in two additional genetic backgrounds using the transparent strain 4DS2341-94 (serotype 4) and EF3030 (serotype 19F), which induce mucosal and lung colonization, respectively (van Ginkel et al., 2003). All four deletion mutant strains displayed greatly reduced in vitro growth rates even in nutrient-rich culture medium (Fig. 1a; Giefing et al., 2008). The mean mass doubling times increased in all mutant strains compared with the corresponding parent wild-type, and were 124 versus $62 \mathrm{~min}$ for TIGR4, 82 versus $56 \mathrm{~min}$ for 6B PJ1324, 109 versus 76 min for 4DS2341-94 and 85 versus 58 min for EF3030. We did not observe any significant difference in terms of autolysis, either in the rate or in the level to which they returned, as all strains, mutant and wildtype, declined to similar optical density values after reaching stationary phase. In vivo these strains can no longer cause disease in mouse models at a very high challenge dose (Giefing et al., 2008; data not shown).

Our attempts to delete PcsB in the D39 and R6 strains were not successful, suggesting that $p c s B$ is indeed essential in these strains. However, based on the successful generation of deletion mutants in four unrelated strains, we concluded that PcsB is not essential for all pneumococcal strains, but many genetic backgrounds, notably recent field isolates, tolerate the absence of PcsB in vitro.

Since addition of recombinant PcsB to the growth medium could not restore the wild-type phenotype in vitro (data not shown), we complemented the $\Delta p c s B$ strain in the $6 \mathrm{~B}$ background with plasmid pMU1328 carrying $p c s B$ in order to connect the observed phenotype unambiguously to the absence of PcsB. We observed that $\triangle p c s B$ strains were hampered in competence, showing approximately 200 -fold lower transformation efficiency than the wild-type strain. Nonetheless, plasmid pMU-pcsB complemented $\Delta p c s B$ cells and restored the growth rate to the level of wild-type cells, while transformation with the empty plasmid was ineffective (Fig. 1b). There was a slight and not significant increase in the growth rate of wild-type cells transformed with pMU-PcsB. Importantly, the expression of PcsB at the protein level in the culture supernatant was similar in the wild-type and complemented strains (Fig. 1c).

Previously we reported an increased antibiotic sensitivity of $\Delta p c s B$ cells in the TIGR4 background (Giefing et al., 2008). Here we extended this analysis to $\triangle p c s B 6 \mathrm{~B}$ cells and confirmed our previous findings obtained with the TIGR4 strain. The increased sensitivity of $\Delta p c s B$ strains relative to the corresponding parent wild-type, which was most pronounced towards antibiotics that target peptidoglycan synthesis, was almost completely eliminated by extrachromosomal PcsB expression (Fig. 2a).

Similarly to other phenotypic changes, the severe defect in septum placement and cell separation, observed by electron microscopic analysis of gene deletion mutant cells (Giefing et al., 2008), was reversed by transformation with the pMU-pcsB plasmid, but not with the empty plasmid. Successfully complemented cells again displayed the typical wild-type diplococcus phenotype with regular septum formation (Fig. 2b).

\section{Nucleoid partition is not affected in PcsB null mutants}

To assess whether DNA duplication and segregation into daughter cells are affected in $\Delta p c s B$ cells, we determined the DNA content of growing cultures by propidium iodide staining combined with flow cytometric analysis (Fig. 3a). DNA staining of wild-type cells revealed two distinct populations, represented by two sharp peaks. The left peak with lower intensity corresponded to non-dividing cells, based on its abundance in stationary phase, while the right peak with higher intensity represented dividing cells with duplicated DNA content. In pcsB deletion mutant cells, instead of two sharp peaks, we observed one broad peak in growing cultures that extended to a higher intensity on the fluorescence scale, suggesting that several DNA duplication events occurred in the cell clusters. Immunofluorescent staining with DAPI revealed that each cell visualized by anti-PspA antibodies contained a similar amount of DNA (Fig. 3b).

The two observations together suggested that the cell separation defect in $\triangle p c s B$ cells occurred after DNA duplication.

\section{PcsB does not lyse peptidoglycan in vitro}

Based on the observed cluster formation and the presence of a CHAP domain, PcsB has been suggested to be involved in peptidoglycan metabolism (Ng et al., 2004; Reinscheid et al., 2001). Therefore, we attempted to detect the cell wall hydrolytic activity of pneumococcal PcsB in in vitro 
(a)
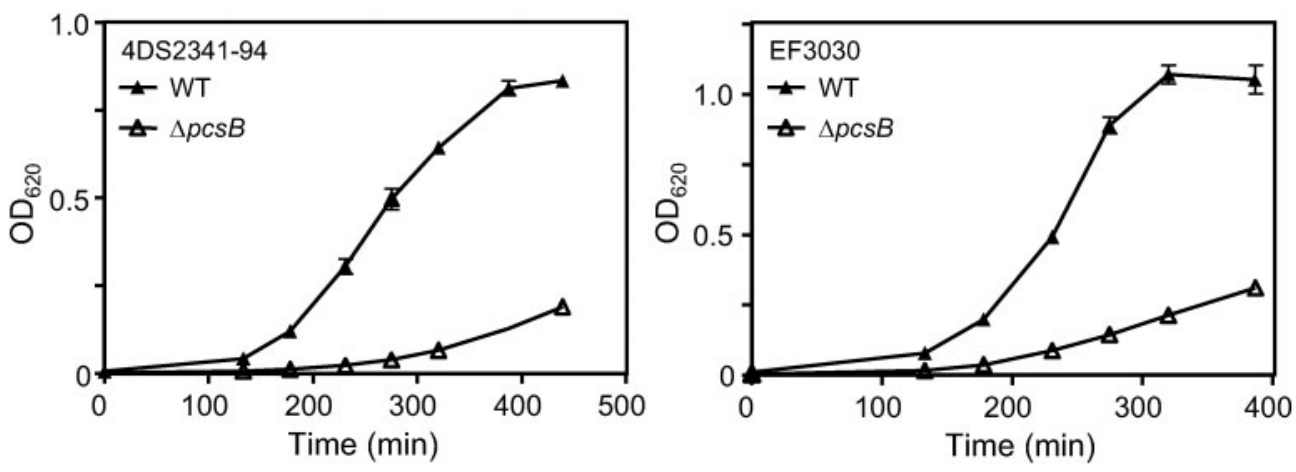

(b)

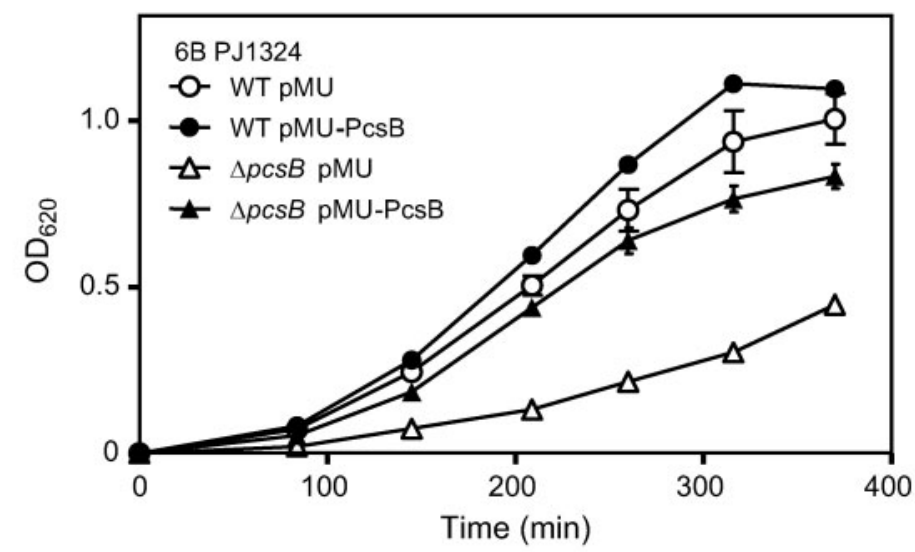

(c)

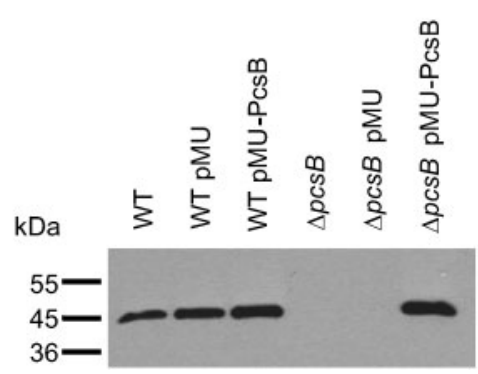

Fig. 1. Wild-type growth rate is restored upon complementation of PcsB in $\Delta p c s B$ cells. (a) In vitro growth of 4DS2341-94 (left panel) and EF3030 (right panel) wild-type and corresponding $\Delta p c s B$ strains. (b) In vitro growth of PJ1324 (serotype 6B) wildtype and $\Delta p c s B$ strains transformed with either empty $\mathrm{pMU}$ or pMU-PcsB. (c) Western blot analysis of culture supernatants (overnight culture) of wild-type and pcsB mutant PJ1324 cells using PcsB-specific antibodies.

biochemical assays in a way similar to that described for the group B streptococcal PcsB homologue (Reinscheid et al., 2001).

We generated recombinant proteins corresponding to the full-length $\mathrm{PcsB}$, as well as its $\mathrm{N}$ - or C-terminal part containing the coiled-coil or CHAP domain, respectively (Fig. 4a, b). First we used an in-gel zymogram assay according to Lepeuple et al. (1998). SDS-treated pneumococcal cells were embedded as substrate in a polyacrylamide gel, and pneumococcal lysate, culture supernatant fractions or recombinant full-length PcsB were separated by
SDS-PAGE. After renaturation, lytic bands were detected by methylene blue staining. We observed only one band with an approximate molecular mass of $36 \mathrm{kDa}$ (probably corresponding to the major autolysin LytA). However, no activity at the expected size of PcsB $(43 \mathrm{kDa})$ in lysate or culture supernatant fractions or with recombinant PcsB-FL was observed (Fig. 4c). Lysate and culture supernatants of $\triangle p c s B$ strains displayed the same pattern of lytic bands as the corresponding wild-type cells (data not shown).

Next, we set up an assay according to Zhou et al. (1988) for the detection of the dye release from RBB-stained 


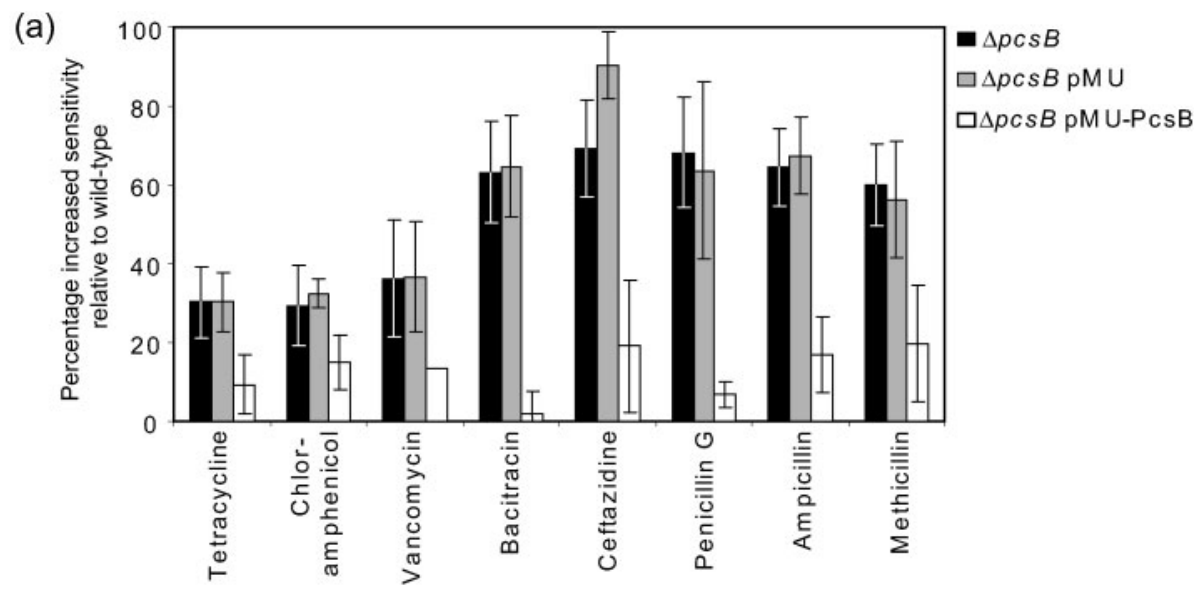

(b)

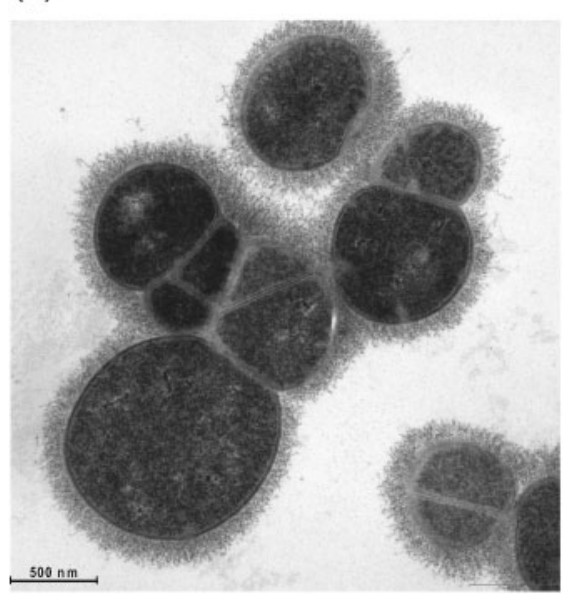

$\Delta p c s B \mathrm{pMU}$

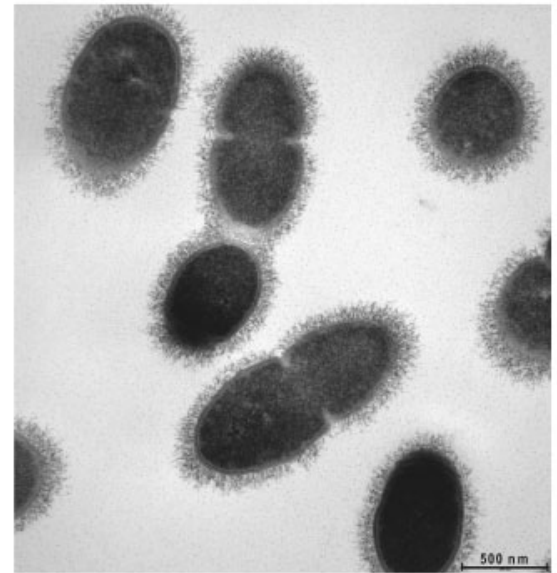

$\Delta p c s B$ pMU-PcsB

Fig. 2. Complementation by extrachromosomal expression of $p c s B$ restores wild-type antibiotic sensitivity and cell morphology. (a) Antibiotic sensitivity was determined by the disc-diffusion method in three independent experiments in PJ1324 $\Delta p c s B$ cells or the same cells transformed with either empty pMU or pMU-PcsB. The average increase in the diameter of the growth inhibition zone (increase in sensitivity) was expressed as a percentage relative to the wild-type. Error bars, SD. (b) Transmission electron microscopy of the $\Delta p c s B$ gene deletion strain transformed with either $\mathrm{pMU}$ alone or pMU-PcsB for complementation.

peptidoglycan that would be expected in the presence of peptidoglycan hydrolase activity. We incubated the recombinant PcsB-FL and its $\mathrm{N}$ - and C-terminal subdomains with the stained peptidoglycan substrate and measured the amount of dye release in the supernatant after incubation. No significant dye release was observed with any of the samples (data not shown).

\section{PcsB has a polar subcellular localization and is excluded from the septum where new peptidoglycan is synthesized}

To determine the subcellular localization of PcsB during cell division and growth, we performed double staining of wild-type pneumococcal cells with antibodies specific for PcsB-FL and FtsZ, the bacterial tubulin homologue that forms the division septum. We did not observe an overlap between FtsZ and PcsB staining in any of the cells. The PcsB signal was excluded from the septal site and the divisome complex and appeared at the cell poles (Fig. 5a, b). The signal intensity was the greatest during exponential growth and was significantly reduced as cells progressed towards the stationary phase, most likely due to increased shedding of PcsB into the culture supernatant (data not shown). When $p c s B$ deletion mutant cells were stained with PcsB-specific antibodies, no immunofluorescence signal was detected (Fig. 5c).

\section{Absence of PcsB induces increased release of proteins to the culture medium}

By analysing culture supernatants of $\Delta p c s B$ strains with SDS-PAGE, we detected differences in the banding pattern relative to the wild-type strain. Certain protein bands, most 
(a)
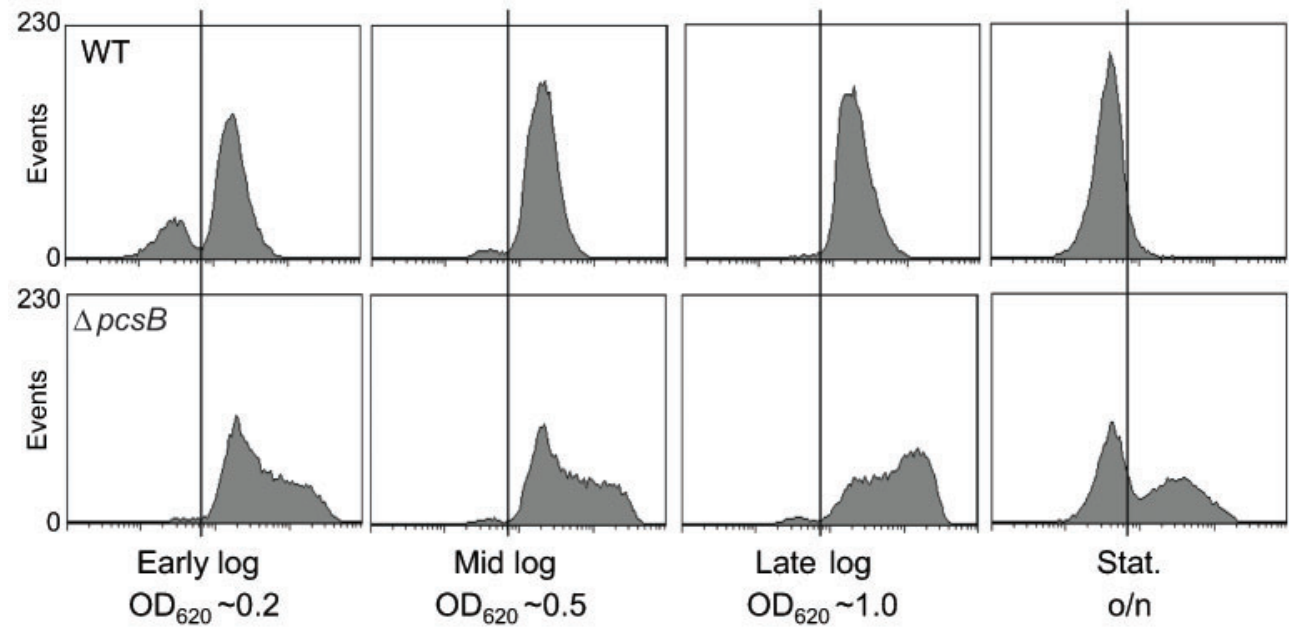

(b)
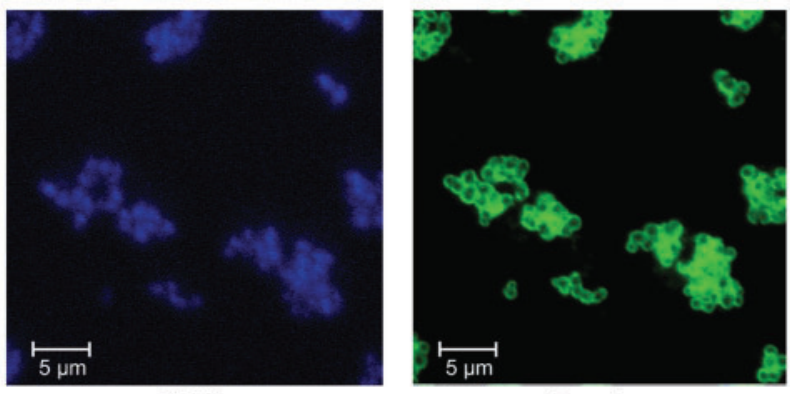

DNA

PspA

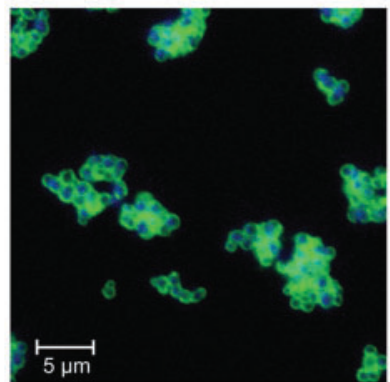

DNA + PspA

Fig. 3. DNA duplication and separation are not affected in $\triangle p c s B$ mutant cells. (a) In wild-type (WT) and $\Delta p c s B$ cells, DNA was stained using propidium iodide and the intensity of the signal was measured in a flow cytometer throughout the progression of the cell cycle as monitored by $\mathrm{OD}_{620}$ readings. All histograms were generated by counting 10000 events. (b) $\Delta p c s B$ mutant cells (strain 4DS2341-94) were double-stained with PspA/Cy2 and DNA/DAPI, as described in Methods, and imaged using a confocal microscope.

prominently an approximately $50 \mathrm{kDa}$ protein, appeared to be more abundant in the culture supernatant of the mutant strain (Fig. 6a). This observation prompted us to further analyse culture supernatants by 2D-PAGE. Mass spectrometric analysis of the $50 \mathrm{kDa}$ protein spot that was the most over-represented in the gene deletion strain supernatant revealed that it was identical to pneumococcal enolase (data not shown). When we sampled the culture supernatant of wild-type and $\Delta p c s B$ strains at multiple time points during growth and analysed them by Western blotting, we observed strong enrichment of enolase in the culture supernatant of the mutant strain that was most intense in the stationary phase (Fig. 6b). The most straightforward explanation for the appearance of enolase in the extracellular space would be the increased fragility of $\Delta p c s B$ cells. However, the selective appearance of certain protein bands did not support bacterial lysis as an explanation.

To investigate the selectivity of protein release, we compared the amounts of a selected set of pneumococcal proteins in wild-type and $\Delta p c s B$ strains. We analysed the culture supernatant fractions of wild-type and deletion mutant pneumococcal cells from the early exponential to the late stationary growth phase by Western blotting with antibodies generated against different pneumococcal proteins available in our laboratory (Giefing et al., 2008). Out of the nine proteins analysed, four were present in increased amounts in the PcsB gene deletion strain, namely SP0107, a LysM domain protein, SP0564 (hypothetical protein), StkP (SP1732) and MalX (maltose/maltodextrin ABC transporter, SP2108) (Fig. 6c). SP0107 and MalX possess predicted signal peptides, the latter with a lipoprotein attachment motif. StkP and SP0564 are not predicted to be secreted by in silico analysis; however, we have reported surface localization of StkP previously (Giefing et al., 2010). Two cell wall proteins with an LPXTG anchor motif, SP0082 and SP0498 (putative endo$\beta$-N-acetylglucosaminidase), the choline-binding cell wall protein PspA and the membrane lipoprotein PsaA were not present in increased amounts in culture supernatants 
(a)
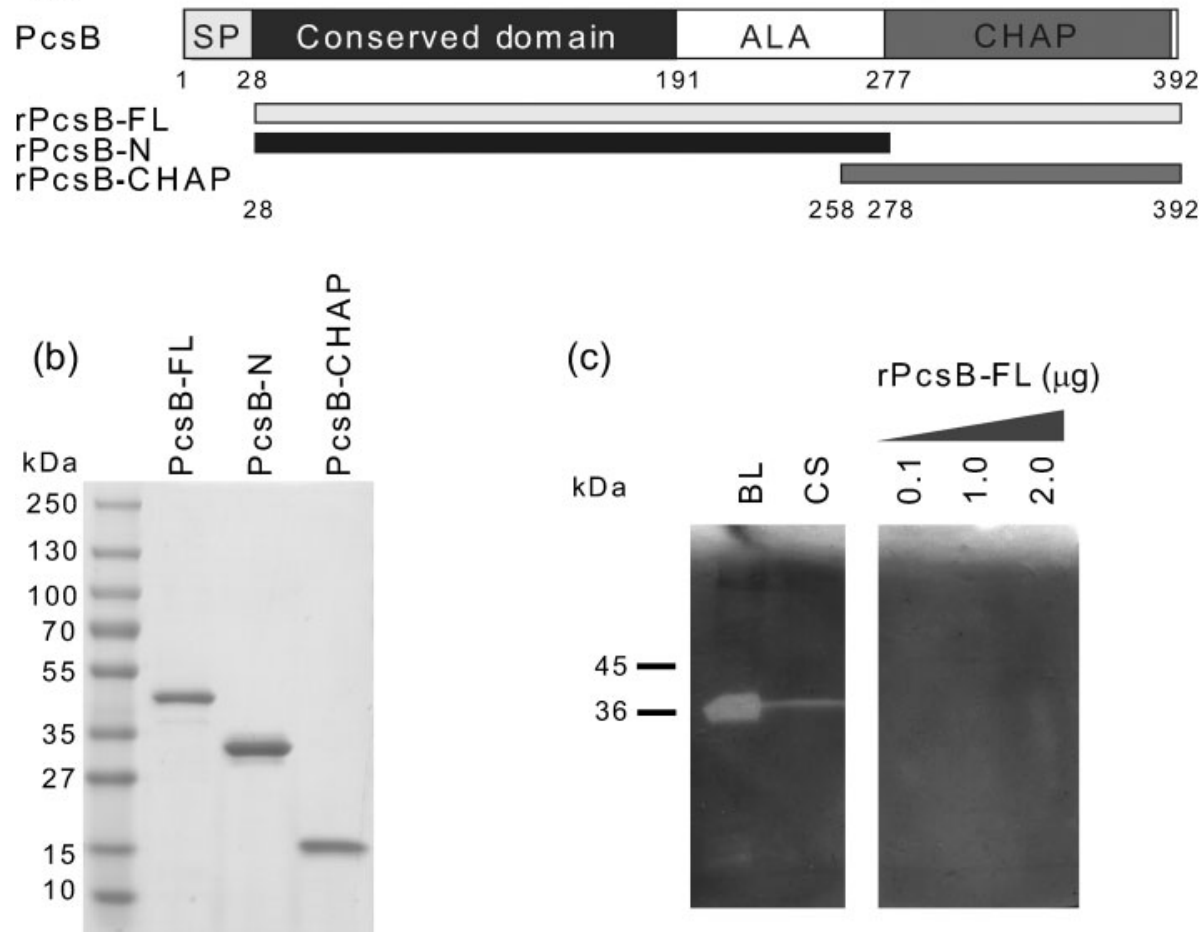

Fig. 4. Lack of lytic activity of PcsB with peptidoglycan. (a) PcsB is composed of four major domains: a signal peptide (SP), a conserved N-terminal domain of unknown function, an alanine-rich region (ALA) and a C-terminal CHAP domain. Recombinant PcsB subdomain proteins expressed in this study are displayed below the full-length PcsB: PcsB-FL (amino acids 28-392), PcsB-N (amino acids 28-278) and PcsB-CHAP (amino acids 258-392). (b) Coomassie blue-stained SDS-PAGE of purified recombinant PcsB variants used in this study. (c) Bacterial lysate (BL) and culture supernatant (CS) from the TIGR4 wild-type strain, as well as recombinant PcsB-FL, were resolved on a cell-gel containing SDS-treated TIGR4 cells and assayed for their lytic activity on the embedded material.

of pcsB-deleted cells relative to wild-type cells. Cytoplasmic pneumolysin was not released to the supernatant, and no difference in expression of this protein between wild-type and mutant strains was observed (data not shown).

Analysis of total lysate fractions (cell-bound proteins) for the same antigens revealed no increased expression for these proteins, except for the LysM domain-containing SP0107 (Fig. 6d and data not shown).

\section{Absence of PcsB results in increased expression of genes encoding LysM domain proteins}

Based on the above observations, we wanted to learn whether compensation mechanisms to overcome the defect of $p c s B$ deletion could be observed at the transcriptional level as well. We performed microarray analysis of bacteria cultivated under the same conditions used for Western blot analysis to screen for differences in mRNA levels between wild-type and gene deletion mutant cells (data not shown). Genes with altered transcription (decreased or increased at least twofold) were further analysed and confirmed by qRT-PCR using RNA from wild-type and $\Delta p c s B$ strains in two different genetic backgrounds (TIGR4 and PJ1324). The most striking difference was the greatly increased transcript levels for two LysM domain proteins, SP0107 and SP2063, which were $>14$-fold and $>25$-fold increased in the TIGR4 strain and the 6B isolate, respectively (based on three independent biological replicates of qRT-PCR analysis). Otherwise, there were a few genes that were affected in both strains, although to a lower extent than the LysM protein genes (Table 1). These included upregulated genes encoding the rrg pilus islet (SP0461-468), a phosphate transport regulatory protein (SP2088), stress proteins GrpE and $10 \mathrm{kDa}$ chaperonin (SP0516, SP1907), and an ATP-dependent Clp protease (SP0338), and downregulated genes encoding translation initiation factor IF-1 (SP0232) and transcriptional regulator GntR (SP1714), as well as three ABC transporter proteins (SP1715, SP1869 and SP1872).

These results suggest that there is a very selective change in transcription as a consequence of the lack of PcsB. 
(a)

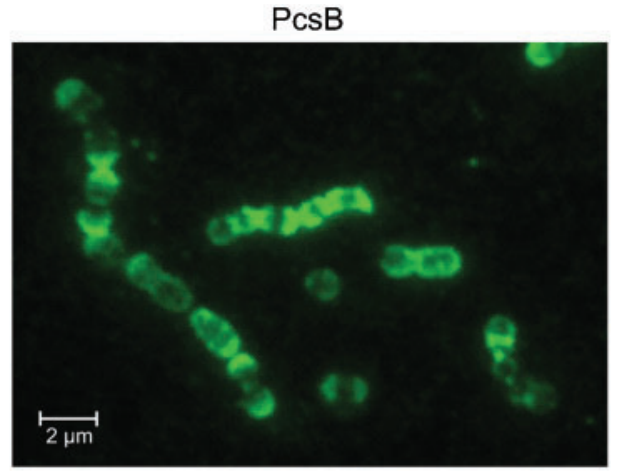

(b)
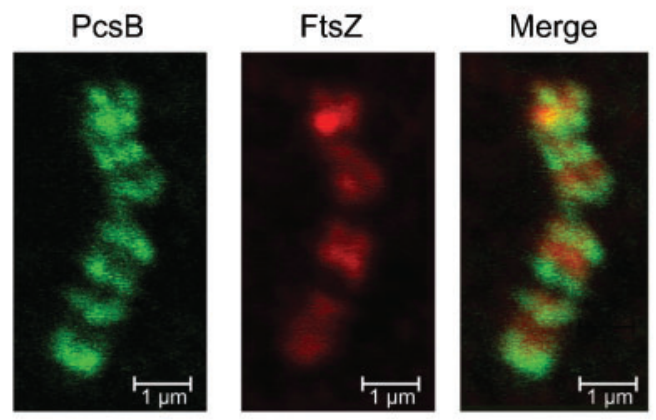

(c)

DAPI
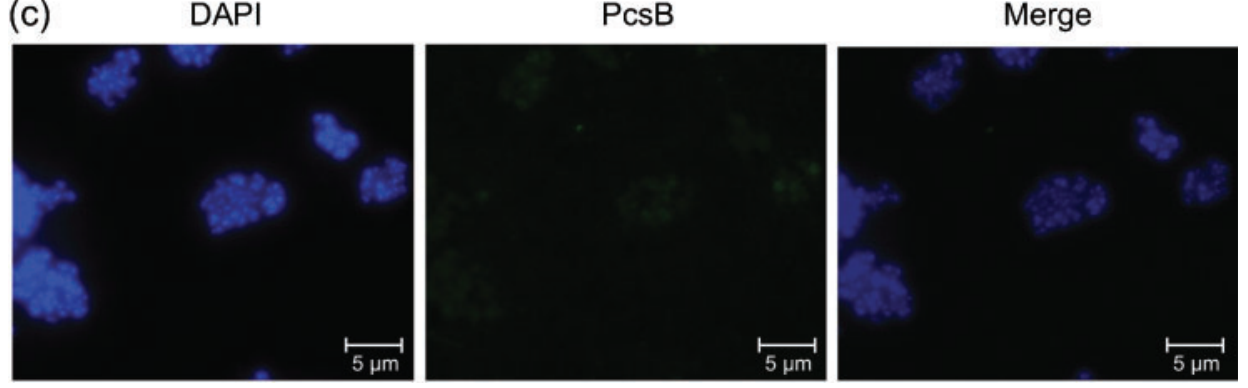

Fig. 5. PcsB is excluded from the site of septal cell wall synthesis. (a, b) Pneumococcal cells (4DS2341-94 wild-type strain) were double-stained with anti-PcsB rabbit serum/Cy2-labelled anti-rabbit IgG and anti-FtsZ mouse serum/Texas Red-labelled anti-mouse lgG. The merged picture shows the overlay of PcsB and FtsZ staining. (c) $\Delta p c s B$ mutant cells (strain 4DS2341-94) were double-stained with PcsB/Cy2 and DNA/DAPI, as described in Methods, and imaged using a confocal microscope. The merged picture shows the overlay of PcsB and DAPI staining.

\section{DISCUSSION}

PcsB in S. pneumoniae and in other streptococcal species has been suggested to have a critical role in cell division. There is a controversy, however, as to whether $p c s B$ null mutant pneumococcal cells are viable or not. PcsB has been found to be essential in the D39 and R6 pneumococcal strains in several studies (Barendt et al., 2009; Ng et al., 2003, 2004, 2005); similarly, we were unable to delete $p c s B$ in these genetic backgrounds (this study). However, we reported recently the successful generation of $p c s B$ null mutants in two genetic backgrounds (TIGR4 and a recent serotype 6B human isolate) (Giefing et al., 2008). Second site (suppressor) mutations have been implicated as an explanation for the viability of these strains (Barendt et al., 2009). In this study we generated additional pneumococcal $p c s B$ mutants in two more genetic backgrounds using the 4DS2341-94 (serotype 4) and EF3030 (serotype 19F) strains (van Ginkel et al., 2003). All four pcsB deletion mutant strains displayed the same cluster-forming phenotype, altered septum formation and retarded in vitro growth, also described for PcsB-underexpressing R6 strains (Ng et al., 2004) and pcsB knockout cells generated in other streptococcal species (Chia et al., 2001a; Reinscheid et al., 2001). Similarly to previous reports (Barendt et al., 2009), we also detected no changes in the transcript levels of the proximal MreCD proteins in our $\Delta p c s B$ mutant strains (based on microarray and Northern blot analysis; data not shown).

In order to directly relate these defects to the absence of PcsB, we complemented one of these mutants by 
(a)

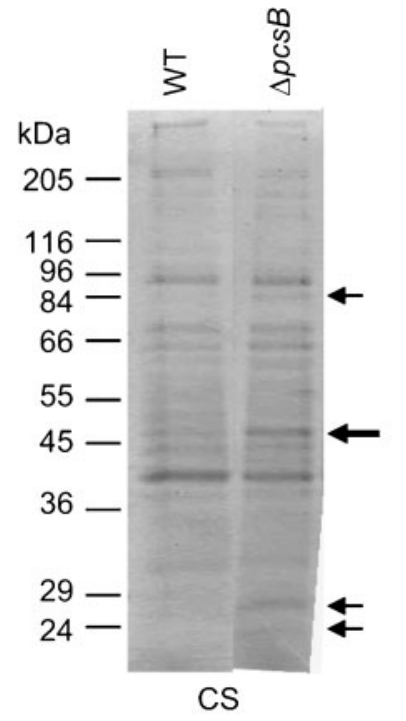

(b)

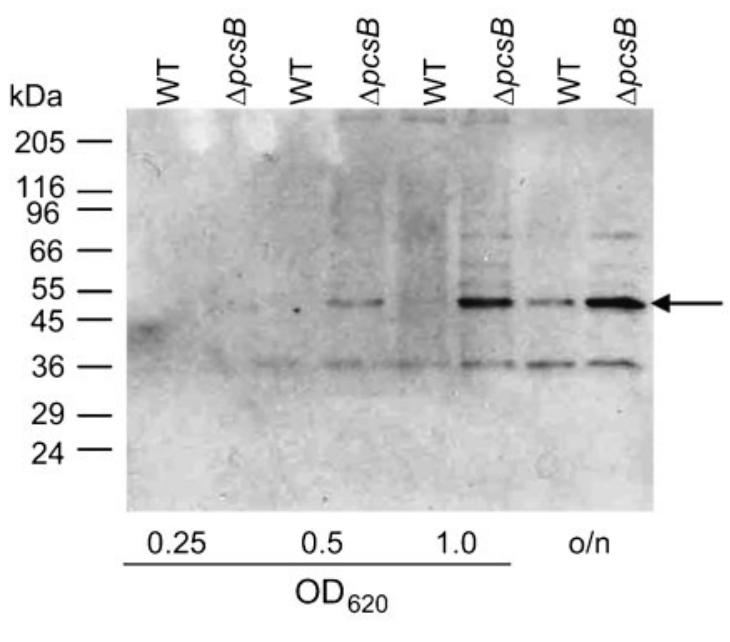

(c)

)

$\mathrm{kDa}$

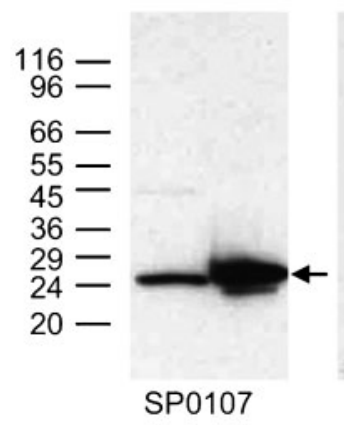

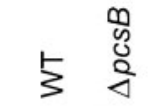

$\begin{array}{r}0 \\ 0 \\ 58 \\ \hline\end{array}$

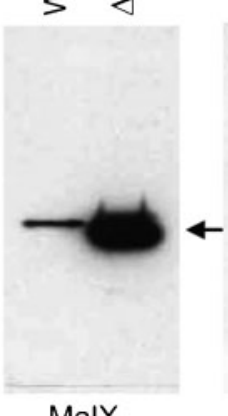

MalX
$5 \stackrel{\substack{0 \\ 0 \\ 2}}{3}$

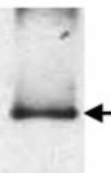

4

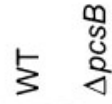
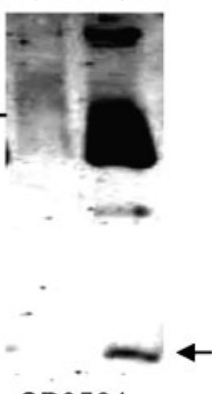

StkP

SP0564

(d)
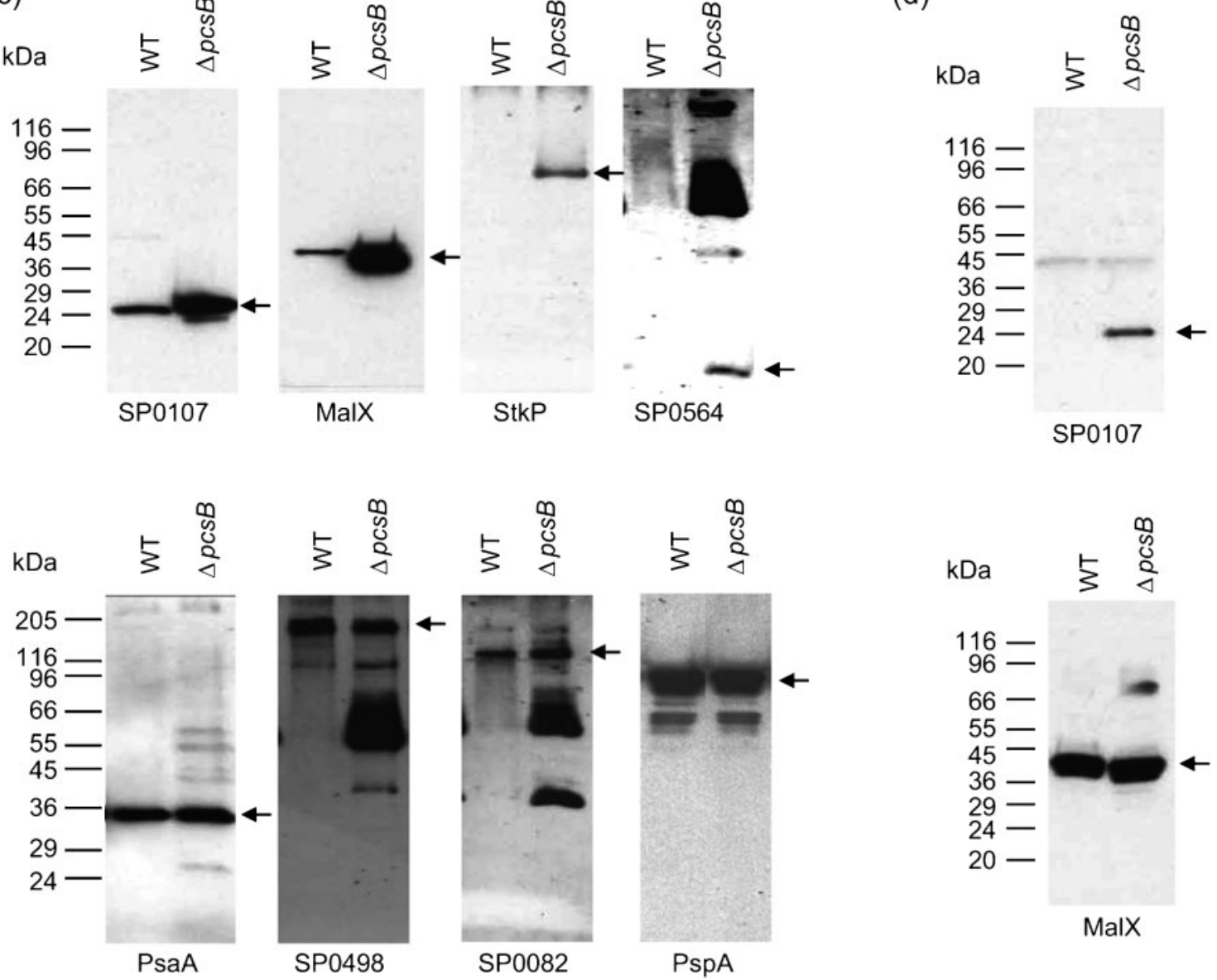

Fig. 6. $\Delta p c s B$ cells display enhanced protein release in vitro. (a) Culture supernatants (CS) of PJ1324 wild-type (WT) and $\Delta p c s B$ cells were separated by SDS-PAGE and stained with Coomassie brilliant blue. (b) Culture supernatants of TIGR4 wild-type and $\triangle p c s B$ cells at different growth stages, based on $\mathrm{OD}_{620}$ readings, were analysed in Western blots using enolase-specific rabbit serum. (c, d) Western blot analysis of culture supernatants from $2 \times 10^{6}$ cells (c) and bacterial lysates (approximately $10^{8}$ cells) (d) of TIGR4 wild-type and $\triangle p c s B$ cells in the exponential growth phase using antibodies specific for the proteins indicated under each panel. Antisera generated against SP0498, SP0082 and SP0564 showed reactivity with unknown proteins that displayed enhanced secretion in mutant strains. The band corresponding to the respective protein is indicated by an arrow to the right of each panel. 
Table 1. Changes in relative transcript amounts due to the absence of PcsB

Genes were selected for real-time PCR analysis based on microarray data. Values represent fold-changes in the $\triangle p c s B$ strains relative to the wild-type strains, shown as the mean of three independent experiments. Only genes downregulated or upregulated at least twofold in the $\Delta p c s B$ strain are indicated. ND, Not determined.

\begin{tabular}{|llcc|}
\hline ORF & \multicolumn{1}{c}{ Gene product } & TIGR4 & 6B \\
\hline SP0107 & LysM domain protein & 14.2 & 26.4 \\
SP2063 & LysM domain protein & 14.8 & 33.6 \\
SP0461 & Transcriptional regulator RlrA & 2.0 & 11.0 \\
SP0462 & LPXTG protein RrgA & 3.4 & 4.8 \\
SP0463 & LPXTG protein RrgB & 3.8 & ND \\
SP0464 & LPXTG protein RrgC & 4.3 & 8.3 \\
SP0467 & Sortase, SrtC & 1.4 & 4.4 \\
SP0468 & Sortase, SrtD & 2.5 & 5.1 \\
SP0338 & ATP-dependent Clp protease, ATP-binding & 13.8 & 2.6 \\
SP0516 & Heat-shock protein GrpE & 10.0 & 2.7 \\
SP1907 & Chaperonin, 10 kDa & 2.8 & 2.6 \\
SP2026 & Alcohol dehydrogenase, iron-containing & 2.3 & 2.2 \\
SP2088 & Phosphate transport system regulatory protein & 7.3 & 18.9 \\
SP2216 & PcsB & -234769 & -268817 \\
SP0232 & Translation initiation factor IF-1 & -3.7 & -2.1 \\
SP1714 & Transcriptional regulator, GntR family & -3.3 & -3.0 \\
SP1715 & ABC transporter, ATP-binding protein & -2.5 & -2.8 \\
SP1869 & Iron-compound ABC transporter, permease & -7.4 & -2.7 \\
SP1872 & Iron-compound ABC transporter, iron- & -5.9 & -2.8 \\
& compound-binding & & \\
\hline
\end{tabular}

transformation with a plasmid carrying $p c s B$ for extrachromosomal expression of the protein. Indeed, the growth rate, cell morphology and antibiotic sensitivity reverted back to wild-type. Thus we conclude that PcsB is not essential for in vitro viability in all pneumococcal strains, but is important for cell division and separation in S. pneumoniae. D39 and its R6 derivative may lack certain sets of genes needed to survive the lack of PcsB that are present in the majority of pneumococci, especially recent field isolates.

Interestingly, the absence of PcsB resulted in apparent differences in protein abundance in the culture supernatant of wild-type and $p c s B$ null mutants, the most obvious increase being associated with enolase (based on 2D PAGE and MS, as well as Western blotting). Interestingly, an increased amount of enolase was also reported in $p c s B$ deletion strains of $S$. agalactiae (Reinscheid et al., 2003). In addition to its catalytic function in the glycolytic pathway, enolase has been found to be an important virulence factor and to be regulated by HtrA, a surface protease involved in the processing and maturation of extracellular proteins (Bergmann et al., 2005; Kolberg et al., 2006). DhtrA mutants in S. mutans display enhanced accumulation not only of enolase but also of PcsB (Biswas \& Biswas, 2005). The accumulation of certain proteins in the supernatant of $\Delta p c s B$ strains seems to be related to their anchoring mechanism: while LPXTG-anchored proteins such as SP0082 or SP0498, or choline-binding proteins such as PspA, were not enriched in the $\Delta p c s B$ strains, other proteins that are probably more loosely attached to the cell wall such as MalX and SP0107, were found at higher concentrations in the supernatant, suggesting micro-changes in the cell wall due to the lack of PcsB.

Based on the strikingly similar morphology of PBP3 (dacA) and $p c s B$ deletion strains we determined the localization of PcsB within the bacterial cell (Schuster et al., 1990; Morlot et al., 2004). By immunofluorescence studies we discovered that PcsB, similarly to PBP3, also mainly localizes at the cellular poles of dividing cells and is excluded from the septal site. In in vitro protein-binding assays using recombinant proteins we did not detect an interaction of PcsB with any of the cell division proteins we tested: FtsZ, FtsA, PBP1b, PBP2a, PBP2x, PBP3 or MreC (data not shown). Despite these parallels to $\mathrm{PBP} 3$, no changes in peptidoglycan composition were detected in $p c s B$ mutants (Barendt et al., 2009; Reinscheid et al., 2003), while altered muropeptide composition is characteristic of PBP3 mutants (Severin et al., 1992).

Interestingly, LytB, a putative endo- $\beta$ - $N$-acetylglucosaminidase, also accumulates at the cell poles and has been shown to be important for daughter cell separation (De Las Rivas et al., 2002). In contrast to LytB, addition of recombinant PcsB to the growth medium could not restore the wild-type phenotype (data not shown), as described for the cell-chaining phenotype of $\Delta l y t B$ strains (De Las Rivas et al., 2002). Thus the inside-to-outside path of PcsB seems to be important for its proper function.

Similarly to others' attempts to associate hydrolytic activity with PcsB in group B streptococci (Reinscheid et al., 2001, 2003), we also failed to detect any peptidoglycan lytic activity 
in vitro either by an in-gel assay or in solution by dye release. CHAP domains are often found in association with other domains that cleave peptidoglycan, and it has been proposed that some members of the CHAP superfamily have lost their amidase activity but still possess a substrate-binding function, e.g. the glucan-binding capacity of $\mathrm{GbpB}$ from $S$. mutans (Rigden et al., 2003). Our data do not exclude the possibility that $\mathrm{PcsB}$ exerts a localized hydrolase activity in vivo that requires an interaction with other proteins. It is interesting in this context that DivIVA, which is important for cell polarity (reviewed in Errington et al., 2003), interacts with PcsB (Fadda et al., 2007). Thus PcsB might be recruited to the cell pole by interaction with DivIVA and thereby exert a localized hydrolytic function that is only active in cooperation with other proteins as soon as the cell division and cell wall synthesis machinery is moved on to the new equatorial sites.

The role of PcsB in peptidoglycan metabolism is further strengthened by our observation that LysM domain-containing proteins, SP0107 and SP2063, were highly overexpressed in $\Delta p c s B$ strains created in two different genetic backgrounds. Interestingly, these genes were also identified as the most upregulated transcripts in a PcsB-underexpressing R6 strain (Barendt et al., 2009). Similarly to the earlier study, the transcriptional changes were otherwise restricted to a small set of genes. We did not observe changes in other genes of the VicRK regulon, such as $l y t B$, which were described elsewhere (Barendt et al., 2009; Ng et al. 2005). However, we also found increased expression of stress response genes, although different ones, namely chaperonin SP1907 and heat-shock protein GrpE instead of groEL and groES, confirming an overall stressed condition of the mutants. Interestingly, we found the pilus islet overexpressed in our piliated strains, possibly pointing towards a link between the $p c s B$ regulatory network and pilus regulation.

To investigate the possible interplay between PcsB and the two LysM domain proteins, which are all regulated by the two-component transcriptional regulator VicR $(\mathrm{Ng}$ et al., 2005), we attempted to generate deletion mutants of SP0107 and SP2063; however, we obtained deletion mutants (in two different backgrounds) only for the latter. $\Delta s p 2063$ strains did not exhibit altered expression of PcsB or SP0107, or any growth or morphological defect observed in $\triangle p c s B$ strains (unpublished data). Vega Masigniani and co-workers recently presented evidence that deletion of SP0107 (spr0096) leads to overall thickening of the cell wall, which points to a role in peptidoglycan hydrolysis (7th International Symposium on Pneumococci and Pneumococcal Diseases, Tel Aviv, Israel, March 16 2010). Our data suggest that lack of PcsB might be partially compensated by overexpression of SP2063 and SP0107 to allow growth, although at a greatly reduced rate.

The activity of hydrolases is often specific for a certain peptidoglycan type, the presence or absence of secondary modifications, high-molecular-weight peptidoglycan or small fragments (Vollmer et al., 2008). Based on our observations and data from other laboratories we suggest a model according to which PcsB is recruited by DivIVA to the future cell pole to generate, through micro-changes in peptidoglycan, the substrate(s) for LytB, LysM domain proteins or other hydrolases that then fulfil their role in cell separation.

\section{ACKNOWLEDGEMENTS}

The authors would like to thank Manfred Berger, Barbara Maierhofer, Dieter Gelbmann and Josef Gotzmann for technical help; Birgitta Henriques-Normark (Swedish Institute for Infectious Disease control), David Briles (University of Alabama) and Eddie Ades (Centers for Disease Control) for providing bacterial strains; and Sven Hammerschmidt (Ernst-Moritz-Arndt-Universität Greifswald) for antiserum. The authors declare a potential conflict of financial interest as employees of Intercell AG, a biotechnology company. The authors have no other competing financial interests.

\section{REFERENCES}

Barendt, S. M., Land, A. D., Sham, L. T., Ng, W. L., Tsui, H. C., Arnold, R. J. \& Winkler, M. E. (2009). Influences of capsule on cell shape and chain formation of wild-type and pcsB mutants of serotype 2 Streptococcus pneumoniae. J Bacteriol 191, 3024-3040.

Bateman, A. \& Rawlings, N. D. (2003). The CHAP domain: a large family of amidases including GSP amidase and peptidoglycan hydrolases. Trends Biochem Sci 28, 234-237.

Bergmann, S., Rohde, M., Preissner, K. T. \& Hammerschmidt, S. (2005). The nine residue plasminogen-binding motif of the pneumococcal enolase is the major cofactor of plasmin-mediated degradation of extracellular matrix, dissolution of fibrin and transmigration. Thromb Haemost 94, 304-311.

Biswas, S. \& Biswas, I. (2005). Role of HtrA in surface protein expression and biofilm formation by Streptococcus mutans. Infect Immun 73, 6923-6934.

Chan, P. F., O'Dwyer, K. M., Palmer, L. M., Ambrad, J. D., Ingraham, K. A., So, C., Lonetto, M. A., Biswas, S., Rosenberg, M. \& other authors (2003). Characterization of a novel fucose-regulated promoter $\left(\mathrm{P}_{f c s K}\right)$ suitable for gene essentiality and antibacterial mode-of-action studies in Streptococcus pneumoniae. J Bacteriol 185, 2051-2058.

Chia, J. S., Chang, L. Y., Shun, C. T., Chang, Y. Y., Tsay, Y. G. \& Chen, J. Y. (2001a). A 60-kilodalton immunodominant glycoprotein is essential for cell wall integrity and the maintenance of cell shape in Streptococcus mutans. Infect Immun 69, 6987-6998.

Chia, J. S., Lee, Y. Y., Huang, P. T. \& Chen, J. Y. (2001b). Identification of stress-responsive genes in Streptococcus mutans by differential display reverse transcription-PCR. Infect Immun 69, $2493-$ 2501.

De Las Rivas, B., García, J. L., López, R. \& García, P. (2002). Purification and polar localization of pneumococcal LytB, a putative endo- $\beta$ - $N$-acetylglucosaminidase: the chain-dispersing murein hydrolase. J Bacteriol 184, 4988-5000.

Divakaruni, A. V., Baida, C., White, C. L. \& Gober, J. W. (2007). The cell shape proteins MreB and MreC control cell morphogenesis by positioning cell wall synthetic complexes. Mol Microbiol 66, 174-188.

Errington, J., Daniel, R. A. \& Scheffers, D. J. (2003). Cytokinesis in bacteria. Microbiol Mol Biol Rev 67, 52-65.

Fadda, D., Santona, A., D'Ulisse, V., Ghelardini, P., Ennas, M. G., Whalen, M. B. \& Massidda, O. (2007). Streptococcus pneumoniae DivIVA: localization and interactions in a MinCD-free context. J Bacteriol 189, 1288-1298. 
Giefing, C., Meinke, A. L., Hanner, M., Henics, T., Bui, M. D., Gelbmann, D., Lundberg, U., Senn, B. M., Schunn, M. \& other authors (2008). Discovery of a novel class of highly conserved vaccine antigens using genomic scale antigenic fingerprinting of pneumococcus with human antibodies. J Exp Med 205, 117-131.

Giefing, C., Jelencsics, K. E., Gelbmann, D., Senn, B. M. \& Nagy, E. (2010). The pneumococcal eukaryotic-type serine/threonine protein kinase StkP co-localizes with the cell division apparatus and interacts with FtsZ in vitro. Microbiology 156, 1697-1707.

Höltje, J. V. (1995). From growth to autolysis: the murein hydrolases in Escherichia coli. Arch Microbiol 164, 243-254.

Höltje, J. V. \& Tuomanen, E. I. (1991). The murein hydrolases of Escherichia coli: properties, functions and impact on the course of infections in vivo. J Gen Microbiol 137, 441-454.

Kolberg, J., Aase, A., Bergmann, S., Herstad, T. K., Rødal, G., Frank, R., Rohde, M. \& Hammerschmidt, S. (2006). Streptococcus pneumoniae enolase is important for plasminogen binding despite low abundance of enolase protein on the bacterial cell surface. Microbiology 152, 13071317.

Kyburz, A., Raulinaitis, V., Koskela, O., Kontinen, V., Permi, P., Kilpelainen, I. \& Seppala, R. (2010). ${ }^{1} \mathrm{H},{ }^{13} \mathrm{C}$ and ${ }^{15} \mathrm{~N}$ resonance assignments of the major extracytoplasmic domain of the cell shapedetermining protein MreC from Bacillus subtilis. Biomol NMR Assign 4, 235-238.

Lepeuple, A. S., Van Gemert, E. \& Chapot-Chartier, M. P. (1998). Analysis of the bacteriolytic enzymes of the autolytic Lactococcus lactis subsp. cremoris strain AM2 by renaturing polyacrylamide gel electrophoresis: identification of a prophage-encoded enzyme. Appl Environ Microbiol 64, 4142-4148.

Livak, K. J. \& Schmittgen, T. D. (2001). Analysis of relative gene expression data using real-time quantitative PCR and the $2^{-\Delta \Delta C_{\mathrm{T}}}$ method. Methods 25, 402-408.

Mattos-Graner, R. O., Jin, S., King, W. F., Chen, T., Smith, D. J. \& Duncan, M. J. (2001). Cloning of the Streptococcus mutans gene encoding glucan binding protein $\mathrm{B}$ and analysis of genetic diversity and protein production in clinical isolates. Infect Immun 69, 69316941.

Mattos-Graner, R. O., Porter, K. A., Smith, D. J., Hosogi, Y. \& Duncan, M. J. (2006). Functional analysis of glucan binding protein B from Streptococcus mutans. J Bacteriol 188, 3813-3825.

Mclver, K. S., Subbarao, S., Kellner, E. M., Heath, A. S. \& Scott, J. R. (1996). Identification of isp, a locus encoding an immunogenic secreted protein conserved among group A streptococci. Infect Immun 64, 2548-2555.

Morlot, C., Noirclerc-Savoye, M., Zapun, A., Dideberg, O. \& Vernet, T. (2004). The D,D-carboxypeptidase PBP3 organizes the division process of Streptococcus pneumoniae. Mol Microbiol 51, 1641-1648.

Ng, W. L., Robertson, G. T., Kazmierczak, K. M., Zhao, J., Gilmour, R. \& Winkler, M. E. (2003). Constitutive expression of PcsB suppresses the requirement for the essential VicR $(\mathrm{YycF})$ response regulator in Streptococcus pneumoniae R6. Mol Microbiol 50, 1647-1663.

Ng, W. L., Kazmierczak, K. M. \& Winkler, M. E. (2004). Defective cell wall synthesis in Streptococcus pneumoniae R6 depleted for the essential PcsB putative murein hydrolase or the VicR (YycF) response regulator. Mol Microbiol 53, 1161-1175.

Ng, W. L., Tsui, H. C. \& Winkler, M. E. (2005). Regulation of the $p s p A$ virulence factor and essential $p c s B$ murein biosynthetic genes by the phosphorylated VicR (YycF) response regulator in Streptococcus pneumoniae. J Bacteriol 187, 7444-7459.

Osborn, M. J. \& Rothfield, L. (2007). Cell shape determination in Escherichia coli. Curr Opin Microbiol 10, 606-610.

Reinscheid, D. J., Gottschalk, B., Schubert, A., Eikmanns, B. J. \& Chhatwal, G. S. (2001). Identification and molecular analysis of PcsB, a protein required for cell wall separation of group B streptococcus. J Bacteriol 183, 1175-1183.

Reinscheid, D. J., Ehlert, K., Chhatwal, G. S. \& Eikmanns, B. J. (2003). Functional analysis of a PcsB-deficient mutant of group B streptococcus. FEMS Microbiol Lett 221, 73-79.

Rigden, D. J., Jedrzejas, M. J. \& Galperin, M. Y. (2003). Amidase domains from bacterial and phage autolysins define a family of $\gamma$-D, L-glutamate-specific amidohydrolases. Trends Biochem Sci 28, 230234.

Schubert, K., Bichlmaier, A. M., Mager, E., Wolff, K., Ruhland, G. \& Fiedler, F. (2003). P45, an extracellular $45 \mathrm{kDa}$ protein of Listeria monocytogenes with similarity to protein p60 and exhibiting peptidoglycan lytic activity. Arch Microbiol 173, 21-28.

Schuster, C., Dobrinski, B. \& Hakenbeck, R. (1990). Unusual septum formation in Streptococcus pneumoniae mutants with an alteration in the D,D-carboxypeptidase penicillin-binding protein 3. J Bacteriol 172, 6499-6505.

Severin, A., Schuster, C., Hakenbeck, R. \& Tomasz, A. (1992). Altered murein composition in a DD-carboxypeptidase mutant of Streptococcus pneumoniae. J Bacteriol 174, 5152-5155.

Smith, D. J., Akita, H., King, W. F. \& Taubman, M. A. (1994). Purification and antigenicity of a novel glucan-binding protein of Streptococcus mutans. Infect Immun 62, 2545-2552.

Smith, D. J., King, W. F. \& Godiska, R. (2001). Passive transfer of immunoglobulin Y antibody to Streptococcus mutans glucan binding protein B can confer protection against experimental dental caries. Infect Immun 69, 3135-3142.

Stewart, G. C. (2005). Taking shape: control of bacterial cell wall biosynthesis. Mol Microbiol 57, 1177-1181.

Teng, F., Kawalec, M., Weinstock, G. M., Hryniewicz, W. \& Murray, B. E. (2003). An Enterococcus faecium secreted antigen, SagA, exhibits broad-spectrum binding to extracellular matrix proteins and appears essential for E. faecium growth. Infect Immun 71, 5033-5041.

van Ginkel, F. W., McGhee, J. R., Watt, J. M., Campos-Torres, A., Parish, L. A. \& Briles, D. E. (2003). Pneumococcal carriage results in ganglioside-mediated olfactory tissue infection. Proc Natl Acad Sci U S A 100, 14363-14367.

Vollmer, W., Joris, B., Charlier, P. \& Foster, S. (2008). Bacterial peptidoglycan (murein) hydrolases. FEMS Microbiol Rev 32, 259-286.

Wuenscher, M. D., Köhler, S., Bubert, A., Gerike, U. \& Goebel, W. (1993). The iap gene of Listeria monocytogenes is essential for cell viability, and its gene product, p60, has bacteriolytic activity. J Bacteriol 175, 3491-3501.

Zapun, A., Vernet, T. \& Pinho, M. G. (2008). The different shapes of cocci. FEMS Microbiol Rev 32, 345-360.

Zhou, R., Chen, S. \& Recsei, P. (1988). A dye release assay for determination of lysostaphin activity. Anal Biochem 171, 141-144.

Edited by: $\mathrm{H}$. Ingmer 